\title{
Channel Estimation Using Aperiodic Binary Sequences
}

\author{
C. Tellambura, Y. J. Guo, and S. K. Barton
}

\begin{abstract}
Estimating a channel impulse response using a known aperiodic sequence is considered. The problem can be reduced to minimizing the trace of the inverse of a Toeplitz matrix. An efficient algorithm for computing this trace is developed and optimal binary sequences up to length 32 are found and tabulated. The use of complementary sequences in this context is also investigated. It is shown that the eigenvalues of the autocorrelation matrices of a pair of complementary sequences sum to a known constant.
\end{abstract}

Index Terms - Channel estimation, complementary sequences, equalization, Toeplitz matrices.

\section{INTRODUCTION}

B URST-TRANSMISSION digital communication systems require channel estimation (CE) for optimal receivers and fast startup equalization. Therefore a known training sequence (TS) is transmitted before the data. This letter describes how to find optimum aperiodic sequences. For time-domain channel estimation, training sequences can be classified broadly into two classes: periodic and aperiodic. This will affect how the autocorrelation function (ACF) is defined and the spectral properties of the sequence. Some practical systems (e.g., GSM) use periodic or cyclically extended training sequences. The subject of periodic sequences for $\mathrm{CE}$ has received much attention (e.g., [1], [2], among many others), while the aperiodic case has received scant attention.

\section{Channel Estimation}

\section{A. Performance Measure}

The complex channel impulse response is assumed to be given by the tap vector $\boldsymbol{h}=\left(h_{0}, h_{1}, \cdots, h_{L-1}\right)$ for $L$ taps,

Manuscript received February 18, 1997. The associate editor coordinating the review of this letter and approving it for publication was Prof. H. V. Poor. This work was carried out at the University of Bradford, West Yorkshire, U.K., supported by the U.K. DTI/EPSRC LINK project PC2011 "High Throughput Radio Modem" under EPSRC Grant GR/K 00318 in collaboration with Symbionics Networks Ltd.

C. Tellambura was with the Telecommunications Research Group, Department of Electrical and Electronic Engineering, University of Bradford, West Yorkshire BD7 1DP, U.K. He is now with the School of Computer Science and Software Engineering, Monash University, Clayton, Vic. 3168, Australia (e-mail: chintha@dgs.monash.edu.au).

Y. J. Guo was with the Telecommunications Research Group, Department of Electrical and Electronic Engineering, University of Bradford, West Yorkshire BD7 1DP, U.K. He is now with Fujitsu Europe Telecom R\&D Center Ltd. (FTRC), Stockley Park, Uxbridge UB11 1AB, U.K. (e-mail: Y.Guo@fujitsu.co.uk).

S. K. Barton was with the Telecommunications Research Group, Department of Electrical and Electronic Engineering, University of Bradford, West Yorkshire BD7 1DP, U.K. He is now with the Institute of Integrated Information Systems, School of Electronic and Electrical Engineering, University of Leeds, Leeds, LS2 9JT, U.K. (e-mail: S.Barton@elec-eng.leeds.ac.uk).

Publisher Item Identifier S 1089-7798(98)04208-2. which remain constant at least for the duration of the training sequence. $\boldsymbol{h}$ is estimated by processing the received signal samples. A sequence $\boldsymbol{b}=\left(b_{0}, b_{1}, \cdots, b_{N-1}\right)$ is initially transmitted for this purpose, where $b_{k} \in\{1,-1\}$. The received signal samples are given by

$$
y_{k}=\sum_{i} h_{i} b_{k-i}+\nu_{k}, \quad k=0,1, \cdots, N-1
$$

which can be written as $\boldsymbol{y}=B \boldsymbol{h}^{t}+\boldsymbol{n}$ where the noise vector $\boldsymbol{n}=\left(\nu_{0}, \nu_{1}, \cdots, \nu_{N-1}\right)^{t}$ and $B$ is an $N \times L$ matrix. The least-squares estimate of the channel vector is [3]

$$
\hat{\boldsymbol{h}}=\left(B^{t} B\right)^{-1} B^{t} \boldsymbol{y}
$$

and $\Phi=B^{t} B$ is the $L \times L$ autocorrelation matrix of the training sequence; the aperiodic $\mathrm{ACF}$ is

$$
\phi(j)=\sum_{i=0}^{N-1-j} b_{i} b_{i+j}, \quad j=0,1, \cdots, N-1
$$

and $\Phi=[\phi(|i-j|)]$. The covariance matrix of the estimate (for white noise with variance $\sigma^{2}$ ) is $\sigma^{2} \Phi^{-1}$, and a performance measure for comparing the quality of different training sequences can be defined as

$$
F=\operatorname{tr}\left(\Phi^{-1}\right) \geq \frac{L}{N}
$$

where $\operatorname{tr}(A)$ (trace) is the sum of the main diagonal elements of matrix $A$. The lower bound (LB) follows from $\operatorname{tr}(\Phi)=N L$ and $\operatorname{tr}(\Phi) \operatorname{tr}\left(\Phi^{-1}\right) \geq L^{2}$.

In [2], least-squares filtering (Wiener filtering) for $\mathrm{CE}$ is considered and optimal binary sequences up to length 22 are found by exhaustive computer search. The search criterion above is used for two cases-nonzero amplitude precursors and zero-amplitude precursors. The resulting sequences offer the best possible signal-to-estimation-error ratio (SER) at the output of the channel estimator.

\section{B. Fast Computation of $\operatorname{tr}\left(\Phi^{-1}\right)$}

Search for optimal training sequences is facilitated if a fast computation method for the trace of the inverse of a Toeplitz matrix can be found. The number of multiplications required for the inversion of an arbitrary nth-order matrix is proportional to $n^{3}$. However, this can be reduced to $n^{2}$ for an $n$ th-order Toeplitz matrix, say, $\Phi$. Moreover, to compute the trace only the elements of the principal diagonal of $\Phi^{-1}$ are required. Furthermore, only $\lfloor(n+1) / 2\rfloor$ of them are required; because if $\left[a_{i j}\right]=\Phi^{-1}$ then $a_{i i}=a_{n-i+1, n-i+1}$. Below is an algorithm designed to exploit these properties using the classical algorithms of Durbin and Trench. 
TABLE I

HeXadecimal Value of Best Aperiodic Binary TS's

\begin{tabular}{cccccc}
\hline \multirow{2}{*}{$L$} & \multicolumn{5}{c}{ TS length $(N)$} \\
\cline { 2 - 6 } & $2 L$ & $2 L+1$ & $2 L+2$ & $2 L+3$ & $2 L+4$ \\
\hline 3 & 4 & 9 & 9 & 12 & 24 \\
4 & 11 & $1 \mathrm{~A}$ & 58 & 53 & A6 \\
5 & 53 & 53 & A6 & CA & 298 \\
6 & $12 \mathrm{E}$ & $\mathrm{CA}$ & 328 & $32 \mathrm{~B}$ & $6 \mathrm{A6}$ \\
7 & $32 \mathrm{~B}$ & 59D & $6 \mathrm{~B} 9$ & CAC & 2474 \\
8 & 3056 & CAC & 2474 & 298B & 14FCD \\
9 & 3257 & 4E5D & D419 & 320D4 & $15 \mathrm{~B} 38$ \\
10 & D419 & 2CBDC & $8 \mathrm{~A} 1 \mathrm{EC}$ & 6A67D & A62F9 \\
11 & $8 \mathrm{~A} 1 \mathrm{EC}$ & 6A67D & 2D111E & 10DCBA & 92A073 \\
12 & 2D111E & 10DCBA & 92A073 & C1772D & 66B7C5 \\
13 & 92A073 & 4D78DD & 12F8EED & 653E6A & AFCD67C \\
14 & 66B7C5 & 653E6A & B350163 & 163A0299 & 2C111DA1 \\
\hline
\end{tabular}

Algorithm 1: Given a real autocorrelation sequence $1=$ $r_{0}, r_{1}, \cdots, r_{n}$ and that $T=\left(r_{|i-j|}\right) \in R^{n \times n}$ is positive definite, the following algorithm [4] computes $y \in R^{n}$ such that $T y=-\left(r_{1}, \cdots, r_{n}\right)^{t}$. Initial conditions: $y_{1}:=-r_{1}$, $\beta:=1$, and $\alpha=-r_{1}$.

$$
\begin{aligned}
& \text { For } \begin{aligned}
& k=1 \cdots, n-1 \\
& \beta:=\left(1-\alpha^{2}\right) \beta \\
& \alpha:=-\left(r_{k+1}+\sum_{i=1}^{k} r_{k+1-i} y_{i}\right) / \beta \\
& \text { For } i=1, \cdots, k \\
& z_{i}:=y_{i}+\alpha y_{k+1-i} \\
& y_{i}:=z_{i} \quad(i=1, \cdots, k) \\
& y_{k+1}:=\alpha .
\end{aligned}
\end{aligned}
$$

This algorithm requires $n^{2}$ multiplications [5].

The following algorithm based on [6] computes the necessary elements on the main diagonal of $T^{-1}$.

Algorithm 2: Given a real autocorrelation sequence $1=$ $r_{0}, r_{1}, \cdots, r_{n}, T_{n}=\left(r_{|i-j|}\right) \in R^{n \times n}$ is positive definite, $A=T_{n}^{-1}$ and $A=\left[a_{i j}\right]$, the following algorithm computes $a_{i i}$ for $2 i \leq n+1$.

Use Algorithm 1 to solve $T_{n-1} y=-\left(r_{1}, \cdots, r_{n-1}\right)^{T}$.

$$
\begin{aligned}
& \gamma:=1 /\left(1+\sum_{k=1}^{n-1} r_{k} y_{k}\right) \\
& a_{11}:=\gamma \\
& \text { For } i=2, \cdots,\lfloor(n-1) / 2\rfloor+1 \\
& \qquad a_{i i}:=a_{i-1, i-1}+\gamma\left(y_{n+1-i}^{2}-y_{i-1}^{2}\right) .
\end{aligned}
$$

These $a_{i i}$ will give $\operatorname{tr}(A)$. Algorithm 2 requires about $2 n$ multiplications after Algorithm 1, which needs $(n-1)^{2}$ multiplications. So the total number of multiplications is about $n^{2}$. Note that this algorithm may also be derived from a triangular decomposition approach [7].

A computer search has been performed to find the best binary nonperiodic sequence for a given number of channel tap weights and sequence length, using the figure of merit $F$, as given in (4). Table I lists the best binary sequences found up to length 32 , represented in hexadecimal. If the sequence length is not a multiple of four, as many zeros as is necessary are added/neglected to form the hexadecimal number (e.g., 1A denotes 010110000 and 58 denotes 0001101000 ). The bits can be mapped to BPSK signals according to: $0 \rightarrow+1$ and $1 \rightarrow-1$. In many cases several sequences achieve the minimum $F$ for given $L$ and $N$. Only one representative is listed in such cases.

The best sequences provide a quality factor that is within a fraction of a decibel of the lower bound. With the number of channel taps held fixed, the quality factor $F$ also tends to become closer to the LB as the TS length increases.

\section{Complementary Sequences}

While the sequences shown in Table I are optimal in the sense of maximizing the output SER, finding long optimal codes is computationally prohibitive. Long complementary sequences offer a solution to this problem since they can easily be obtained by recursive methods and are known to have reasonably flat spectra.

To get an idea about the degree of optimality of complementary sequences, the following figure of merit is defined. Let $\Phi$ be the autocorrelation matrix associated with a TS of length $N$ and $\lambda_{\max }$ be the largest eigenvalue of $\Phi$. The figure of merit is

$$
\eta=\frac{\lambda_{\max }}{N} .
$$

For a perfect TS [i.e., $\phi(j)=N \delta(j)$ where $\delta(j)$ is the impulse function], $\Phi=N I$, where $I$ is the identity matrix, and $\eta=1$. Otherwise, $\eta \geq 1$.

Two sequences $\left\{a_{i}\right\}$ and $\left\{b_{i}\right\}$ of length $N$ form a complementary pair if their aperiodic ACF's (3) satisfy [8]

$$
\phi_{a}(j)+\phi_{b}(j)=2 N \delta(j) .
$$

Therefore, the corresponding correlation matrices satisfy

$$
\Phi_{a}+\Phi_{b}=2 N I .
$$

It follows that eigenvalue pairs, $\lambda_{a}$ and $\lambda_{b}$, of $\Phi_{a}$ and $\Phi_{b}$, respectively, satisfy

$$
\lambda_{a}+\lambda_{b}=2 N .
$$

Thus, for a pair of complementary sequences, pairs of eigenvalues of the associated matrices are themselves complementary. Since the eigenvalues of a correlation matrix are nonnegative, $0 \leq \lambda_{a}, \lambda_{b} \leq 2 N$. That is, $\lambda_{\max } \leq 2 N$ for a complementary sequence. Thus, the figure of merit $\eta \leq 2$. Therefore, complementary sequences perform quite close to the ideal sequences.

\section{CONCLUSIONS}

In this letter a performance measure has been proposed to assess the quality of binary sequence for $\mathrm{CE}$, using the trace of the inverse of its associated autocorrelation matrix. An algorithm for fast computing this trace has been developed and used to find optimal aperiodic codes up to length 32 . For longer lengths, complementary sequence pairs are proposed and it is shown that these codes are quite close to ideal, by reference to a figure of merit which, for a given $N$, is proportional to the largest eigenvalue of the associated autocorrelation matrix. 
Moreover, the autocorrelation matrices of a complementary sequence pair have eigen spectra that sum to a constant.

\section{REFERENCES}

[1] A. Milewski, "Periodic sequences with optimal properties for channel estimation and fast start-up equalization," IBM J. Res. Develop., vol. 27, pp. 426-431, 1983.

[2] S. N. Crozier, D. D. Falconer, and S. A. Mahmoud, "Least sum of squared errors (LSSE) channel estimation," Proc. Inst. Elect. Eng.—pt. F, vol. 138, pp. 371-378, Aug. 1991.
[3] L. L. Scharf, Statistical Signal Processing. Reading, MA: AddisonWesley, 1991.

[4] J. Durbin, "The fitting of time series models," Rev. Inst. Int. Stat., vol. 28, pp. 233-243, 1960.

[5] G. H. Golub and C. F. Van Loan, Matrix Computations. Oxford, U.K.: North Oxford Academic, 1983.

[6] W. F. Trench, "An algorithm for the inversion of finite Toeplitz matrices," J. SIAM, vol. 12, pp. 515-522, 1964.

[7] C. W. Therrien, Discrete Random Signals and Statistical Signal Processing. Englewood Cliffs, NJ: Prentice Hall, 1992.

[8] M. J. E. Golay, "Complementary series," IRE Trans. Inform. Theory, vol. IT-7, pp. 82-87, Apr. 1961. 